\title{
A general formula for event-based stabilization of nonlinear systems
}

\author{
Nicolas Marchand, Sylvain Durand and Jose Fermi Guerrero Castellanos
}

\begin{abstract}
In this paper, a universal formula is proposed for event-based stabilization of general nonlinear systems affine in the control. The feedback is derived from the original one proposed by E. Sontag in the case of continuous time stabilization. Under the assumption of the existence of a smooth Control Lyapunov Function, it is proved that an event-based static feedback, smooth everywhere except at the origin, can be designed so to ensure the global asymptotic stability of the origin. Moreover, the intersampling time can be proved not to contract at the origin. More precisely, it is proved that for any initial condition within any given closed set the minimal inter-sampling time is proved to be below bounded avoiding the infinitely fast sampling phenomena. Moreover, under homogeneity assumptions the control can be proved to be smooth anywhere and the inter-sampling time bounded below for any initial condition. In that case, we retrieve a control approach previously published for continuous time stabilization of homogeneous systems.
\end{abstract}

\section{INTRODUCTION}

The classical discrete time framework of controlled systems consists in sampling the system periodically with a constant time period $T$ and in computing/updating the control every $t_{k}=k \cdot T$. This field, denoted as the time-triggered case, has been widely investigated for linear control systems (see [1] and the references therein), even in the case of delays, sampling jitter and measurement loss [2]. In the case of nonlinear control systems, one way to address a discrete-time feedback is to implement a continuous time control algorithm with a sufficiently small sampling

N. Marchand is with GIPSA-lab laboratory, Control Systems Dept., SySCo team, CNRS-Univ. of Grenoble, ENSE3 BP 46, 38402 St Martin d'Hères Cedex, France nicolas.marchand@gipsa-lab.inpg. fr

S. Durand is with NeCS team, INRIA - GIPSA-lab - CNRS - Univ. of Grenoble, Grenoble, France, sylvain.durandeinrialpes. fr

J.F. Guerrero Castellanos is with Autonomous University of Puebla (BUAP), Faculty of Electronics, Puebla, Mexico, fguerrero@ece.buap.mx 
period [3]. However, this can be constrained by hardware and reducing the sampling period to a level that guarantees acceptable closed-loop performance may be impossible. Other way to tackle this problem is the application of sampled-data control algorithms based on approximate discretetime models [4] which is not a trivial task. Another proposed approach consist to modify a continuous time stabilizing control using a general formula to obtain a redesigned control suitable for sampled-data implementation [5]. Beside these works, sampling was also used for control theory purpose in order to establish an equivalence between controllability and stabilizability for nonlinear systems [6], [7]. However, in these approaches, the control remains time varying in between sampling instants contrary to the case addressed in this paper.

To overcome the complexity of periodic sampling, event-based control has been recently proposed. In this control strategy the control task is executed after the occurrence of an external event (event-triggered) or, as focused in this paper, after the occurrence of an internal event generated by some event function (self-triggered). In this scheme, the inter-sampling time denotes the time interval between two consecutive events. The sampling instants are not necessarily equidistant in time. Let us first consider general nonlinear systems of the form:

$$
\dot{x}=f(x, u)
$$

where $x \in \mathcal{X} \subset \mathbb{R}^{n}, u \in \mathcal{U} \subset \mathbb{R}^{p}$, and $f$ a Lipschitz function vanishing at the origin. For sake of simplicity, we only consider in this paper null stabilization with initial time instant $t_{0}=0$. Event-based feedback usually means a set of two functions:

- an event function $e: \mathcal{X} \times \mathcal{X} \rightarrow \mathbb{R}$ that indicates if one needs $(e \leq 0)$ or not $(e>0)$ to update the control value. Event function $e$ takes the current state $x$ as input and a memory $m$ of $x$ last time $e$ became negative. There is a priori no constraint on the regularity of $e$. Memoryless (that is $e(x))$, time-varying $(e(x, m, t))$ or simply time index depending $(e(x, m, k))$ is not the purpose of the contribution.

- a feedback function $k$. The terms static (depending upon $x$ ) and dynamic (depending upon $x$ and $t$ or $k$ ) will then be used as in the classical frame.

Typical event mechanisms are functions of the state variation (or the output) of the system, like in [8], [9], [10], [11], [12]. Although the event-triggered control is well-motivated and allows to relax the periodicity of computations, only few works report theoretical results about the stability, 
convergence and performance. In [13] for instance, it is proved that such an approach reduces the number of sampling instants for the same final performance. Recent works deal with the problem of scheduling the control task for continuous-time linear systems [13], [14], [15], [16] and discrete-time linear system [17] where stability and some robustness proprieties such as ISS and $\mathcal{L}_{\infty}$-performance are exploited. Furthermore, in [17] a Model Predictive Control scheme is used and the event-triggered policies relax the computationally demanding algorithms. Some of the above contributions do not need memory of the last sampling.

An alternative approach consists in taking $e$ related to the variation of a Lyapunov function and consequently to the state too - between the current state and its value at the last sampling, like in [18], [19], or in taking $e$ related to the time derivative of the Lyapunov function. An important contribution for convergence and stability in the nonlinear case is studied in [20], [21], [22]. Their main contribution is the existence of a minimal inter-sampling time for any bounded initial conditions. The update policy is based on the existence of a Lipchitz stabilizing control law and an ISS-CLF, that is a CLF such that $\frac{\partial V}{\partial x} f(x, k(x+\varepsilon)) \leq-\alpha(\|x\|)+\beta(\|\varepsilon\|)$ where $\alpha$ and $\beta$ are two functions of class $\mathcal{K}_{\infty}$ and $\varepsilon=m-x$ denotes the measurement error. The update is then computed as soon as $\beta(\|\varepsilon\|) \leq \sigma \alpha(\|x\|)$ ensuring that way the strict decrease of the CLF with $0<\sigma<1$. Results are given for general non linear systems, homogeneous systems (quite in the spirit of the second part of this paper) and polynomial systems. However, in these works is assumed that the control is Lipschitz at the origin. Unfortunately it is known that general formulas as treated in this paper can not ensure this strong property. Moreover, the event policy is based on the knowledge of an upper bound of the CLF with respect to state and measurement error, which is not the case of this paper.

The solution of (1) with event-based feedback $(e, k)$ starting in $x_{0} \in \mathcal{X}$ at $t=0$ is then defined as the solution (when it exists) of the differential system:

$$
\begin{aligned}
& \dot{x}=f(x, k(m)) \\
& \left\{\begin{array}{l}
m=x \quad \text { if } e(x, m) \leq 0, x \neq 0 \\
\dot{m}=0
\end{array}\right. \text { elsewhere } \\
& \text { with: } x(0)=x_{0} \text { and } m(0)=x(0)
\end{aligned}
$$

If $f$ is assumed to be Lipschitz, a unique solution in the Caratheodory sense always exists without 
any smoothness assumption on $k$ similarly to [6] with punctual events. However, this solution may not exists for all $t \geq 0$ as shown by example 2 of section II. Let $t \rightarrow x\left(t, x_{0}\right)$ denote this solution. Given an event function $e$, and a feedback $k$ defined as above, for any initial condition $x(t=0)=x_{0}$ it fully defines a sampling set $T_{e, k, x_{0}}:=\left\{t_{0}, t_{1}, t_{2}, \ldots\right\}$ as the set of time instant $t_{0}=0, t_{1}$, etc. (called sampling instants) at which $e$ is negative. The event-based closed-loop solution is therefore defined at least for all positive $t$ in $\left[0, \sup \left(T_{e, k, x_{0}}\right)[\right.$. This interval is closed if $\sup \left(T_{e, k, x_{0}}\right) \in T_{e, k, x_{0}}$. To illustrate this we give in the next section different examples of possible phenomena. This will introduce new notions and definitions given in section III. Section IV is dedicated to the main theorem that extends Sontag's universal formula for feedback stabilization to event-based stabilization.

Notations: In the following, $\mathcal{B}(d, x)$ will stand for the ball of radius $d$ centred at $x$ and $\mathcal{B}(d)$ for the ball of radius $d$ centred at the origin. $x\left(t ; x_{0}, t_{0}, u\right)$ will denote the solution of a differential system starting in $x_{0}$ at $t_{0}$ with control $u$. For sake of simplicity, $u$ will be omitted when trivial and $x\left(t ; x_{0}\right)$ will stand for $x\left(t ; x_{0}, 0\right)$. $\|\cdot\|$ will stand for the classical $L_{2}$ euclidian norm. $\|\cdot\|_{p}$ will stand for the $L_{p}$-norm. For diagonal matrices, the norm will denote the norm of its diagonal taken as a vector.

\section{WHAT CAN HAPPEN WITH EVENT-BASED CONTROL ?}

To illustrate different phenomena that can arise with event-based feedback systems, we consider the simple linear integrator $\dot{x}=u$. All event functions considered in these examples are assumed to be memoryless that is to be just functions of $\mathcal{X}$. Between two sampling instants $t_{i}$ and $t_{i+1}, u$ remains constant so that: $x_{i+1}=x_{i}+\left(t_{i+1}-t_{i}\right) \cdot u, x_{i}$ denoting the value of the state when the $i^{\text {th }}$ event occurs. The following examples illustrate what may happen.

1) Take $k(x)=-x, e(x)=0$ when $|x|=\exp (-\kappa), \kappa \in \mathbb{Z}$ and initial condition $x_{0}=0$. Then $T_{e, k, x_{0}}:=\{0\}$. Now, taking $x_{0}=1$, the solution is $x_{i}=\exp (-i)$, the inter-sampling time is constant (equal to $1-\exp (-1)$ ) and $T_{e, k, x_{0}}:=\{j \cdot(1-\exp (-1)), j \in \mathbb{N}\}$. For both initial conditions trajectories are well-defined for all $t \in[0,+\infty[$.

2) Take $k(x)=-x^{\frac{1}{2}}, e(x)=0$ when $|x|=\frac{1}{\kappa}, \kappa \in \mathbb{Z}$ and initial condition $x_{0}=1$. In that case, the inter-sampling interval $t_{i+1}-t_{i}=\frac{1}{i^{\frac{1}{2}}(i+1)}$. And when $i$ tends to infinity, the sampling interval tends to zero and $t_{i}$ converges to some $\bar{t} \approx 1.86$. The trajectory is then well-defined only for all $t \in[0, \bar{t}[$. 
3) Take $k(x)=-x, e(x)=0$ when $|x|=\frac{1}{\kappa}, \kappa \in \mathbb{Z}$ and initial condition $x_{0}=1$. In that case, one can calculate that $t_{i}=i-\sum_{j=1}^{i} \frac{j-1}{j}$ and $t_{i+1}-t_{i}=\frac{1}{i}$. Hence the inter-sampling time tends to zero although the trajectory is well defined on $[0, \infty[$.

4) Take $k(x)=-x^{3}, e(x)=0$ when $|x|=\exp (-\kappa), \kappa \in \mathbb{Z}$ and initial condition $x_{0}=1$. Then, the inter-sampling time $t_{i+1}-t_{i}=\frac{x_{i+1}-x_{i}}{-x_{i}^{3}}=\exp (2 i) \cdot[1-\exp (-1)]$ and when $i$ tends to infinity, $t_{i+1}-t_{i}$ also tends to infinity. The trajectory is well-defined for all $t \in[0,+\infty[$ as in case 1 .

Consider now the unstable system $\dot{x}=(x+u)^{3}$. The control $u$ being constant between each sampling instant, the solution is the one of a Bernoulli differential system whose solution is:

$$
x_{i+1}=\frac{x_{i}+u}{\sqrt{1-2\left(t_{i+1}-t_{i}\right) \cdot\left(x_{i}+u\right)^{2}}}-u
$$

Then, taking

5) $k(x)=-2 x, e(x)=0$ when $|x|=\exp (-\kappa), \kappa \in \mathbb{Z}$ and initial condition $x_{0}=1$. Then the inter-sampling duration is:

$$
t_{i+1}-t_{i}=\frac{\exp (2 i)}{2} \cdot\left[1-\frac{1}{(2-\exp (-1))^{2}}\right]
$$

and when $i$ tends to infinity, $t_{i+1}-t_{i}$ also tends to infinity. However, the origin of the closed loop system can be proved to be asymptotically stable and the trajectories well-defined on $[0,+\infty[$ for any initial condition.

In cases 1 to 5 , the system can trivially be proved to be globally null-asymptotically stable. Cases 1 show that the sampling set is initial condition dependent. Cases 1 to 4 show that for the same system and initial condition, the sampling can be periodic, contractile or expansile (with a finite or infinite limit) depending upon the event function or the feedback. Case 5 shows the inconsistency of Shannon's sampling theorem in the event-based paradigm since the intersampling duration can infinitely increase even with an open-loop unstable system.

\section{PRELIMINARY DEFINITIONS FOR EVENT-BASED S YSTEMS}

Usually, the set of event instants is of null measure, in the sense that the control is recomputed only at distinct $t$. However, taking $e(x)=0$ for all $x \in \mathcal{X}$ would mean that one recomputes the control at each $x$ and therefore that one applies a classical continuous-time feedback. On the 
sets of non null measure where $e(x)=0$, the solution is understood in the classical sense (with all possible solution existence problems if the field is discontinuous). Elsewhere, the solution can be intended in the Caratheodory sense. To go further on that, we define:

Definition 3.1 (Well-defined event-based control): An event-based control $(k, e)$ will be said well-defined if and only if for any initial condition $x_{0}$ at $t=0$, the solution $t \rightarrow x\left(t ; x_{0}\right)$ exists for all $t \geq 0$.

Property 3.1 (Minimal Sampling Interval - MSI): An event-based control $(k, e)$ will be said to satisfy the Minimal inter-Sampling Interval property (MSI) iff for any initial condition $x_{0}$ at $t=0$, there exists a non zero minimal sampling interval $\underline{\tau}\left(x_{0}\right):=\inf _{i \in \mathbb{N}, t_{i} \in \mathcal{T}\left(x_{0}\right)} t_{i+1}-t_{i}>0$. The aim of this property is to avoid zero inter-sampling time leading to zeno phenomena at finite time as in example 2 or at infinity as in example 3 as explained in the last section. In case the MSI property is satisfied, the control is piecewise constant between each time sample and $x_{i}$, $i \in \mathcal{I} \subset \mathbb{N}$ with $x_{0}:=x(t=0)$ will denote the series of successive state values at which $e$ is negative for a given initial condition $x_{0}$. To this series corresponds naturally the time series $t_{i}$, $i \in \mathcal{I} \subset \mathbb{N}$ with $t_{0}:=0$. It trivially follows (proof is omitted):

Theorem 3.2: An MSI event-based control is well-defined.

This minimal sampling period is useful for implementation purpose but also when the feedback $k$ is discontinuous for robustness purpose [23]. However, it would be more suitable to have such a bound less depending upon the initial condition:

Property 3.3 (Semi-uniformly MSI event-based control): An event-based control $(k, e)$ will be said semi-uniformly MSI iff for any $\delta>0, \underline{\tau}(\delta):=\inf _{i \in \mathbb{N}, t_{i} \in \mathcal{T}\left(x_{0}\right), x_{0} \in \mathcal{B}(\delta)} t_{i+1}-t_{i}>0$

Property 3.4 (Uniformly MSI event-based control): An event-based control $(k, e)$ will be said uniformly MSI iff $\underline{\tau}:=\inf _{i \in \mathbb{N}, t_{i} \in \mathcal{T}\left(x_{0}\right), x_{0} \in \mathcal{X}} t_{i+1}-t_{i}>0$.

Properties 3.1 to 3.4 can be specified adding the term "global" when $\mathcal{X}=\mathbb{R}^{n}$ in opposition to the term "local" that was omitted above for sake of simplicity. Now that the above notions for eventbased controlled systems are appropriately defined, notions like stability, asymptotic stability and stabilizability naturally follow since they rely on the resulting trajectory. The question that arises then is: does a universal formula for uniformly discrete event-based feedback stabilization exist similarly to the continuous time case ? This is the purpose of the next section. 


\section{A UNIVERSAL FORMULA FOR EVENT-BASED STABILIZATION}

In the sequel, the analysis is restricted to systems affine in the control:

$$
\dot{x}=f(x)+g(x) u=f(x)+\sum_{i} g_{i}(x) u_{i}
$$

where $f$ and $g$ are smooth functions with $f$ vanishing at the origin. We assume that a Control Lyapunov Function (CLF) exists for system (5), that is a smooth and positive definite function $V: \mathcal{X} \rightarrow \mathbb{R}$ so that for each $x \neq 0$ there is some $u \in \mathcal{U}$ such that:

$$
\frac{\partial V}{\partial x} f(x)+\frac{\partial V}{\partial x} g(x) u<0
$$

In addition, one may require that $V$ fulfills the small control property [24], that is that for each $\varepsilon>0$ there is some $\mu>0$ such that for any $x$ in the ball $\mathcal{B}(\mu) \backslash\{0\}$, there is some $u$ with $\|u\| \leq \varepsilon$ such that (6) holds. Then, it is known that it is possible to design a feedback control that asymptotically stabilizes the system [24]:

Theorem 4.1 (Sontag's universal formula): Assume that system (5) admits $V$ as CLF. For any real analytic function $q: \mathbb{R} \rightarrow \mathbb{R}$ such that $q(0)=0$ and $b q(b)>0$ for $b \neq 0$, let $\phi: \mathbb{R}^{2} \rightarrow \mathbb{R}$ be defined by $\phi(a, b):=\frac{a+\sqrt{a^{2}+b q(b)}}{b}$ if $b \neq 0$ and zero when $b=0$. Let the feedback $k: \mathcal{X} \rightarrow \mathcal{U}$, smooth on $\mathcal{X} \backslash\{0\}$ be defined by $k_{i}(x):=-b_{i}(x) \phi(a(x), \beta(x)), i \in\{1, \ldots, p\}$ with $a(x):=\frac{\partial V}{\partial x} f(x), b(x):=\frac{\partial V}{\partial x} g(x), \beta(x):=\|b(x)\|^{2}$. Then $k$ is such that for all non zero $x$, $\frac{\partial V}{\partial x} f(x)+\frac{\partial V}{\partial x} g(x) k(x)<0$. Moreover, if the CLF satisfies the so called small control property, then taking $q(b):=b$, the control is continuous at the origin .

The main purpose of this paper is to establish that a universal formula also exists in the eventbased context up to a modification of the original formula proposed by Sontag:

Theorem 4.2: If there exists a CLF for system (5), then the event-based feedback $(e, k)$ defined below is semi-uniformly MSI, smooth on $\mathcal{X} \backslash\{0\}$, and such that:

$$
\frac{\partial V}{\partial x} f(x)+\frac{\partial V}{\partial x} g(x) k(m)<0, \quad x \in \mathcal{X} \backslash\{0\}
$$

where $m$ is defined by (3) and:

$$
\begin{aligned}
k_{i}(x) & :=-b_{i}(x) \delta_{i}(x) \gamma(x) \\
e(x, m) & :=-a(x)-b(x) k(m)-\sigma \sqrt{a(x)^{2}+\theta(x) b(x) \Delta(x) b(x)^{T}}
\end{aligned}
$$


where

- $a(x)$ and $b(x)$ are as in Theorem 4.1,

- $x \rightarrow \Delta(x):=\operatorname{diag}\left(\delta_{1}(x), \delta_{2}(x), \ldots, \delta_{p}(x)\right)$ is a smooth function of $\mathcal{X} \backslash\{0\}$ to $\mathbb{R}^{p \times p}$, positive definite on $\mathcal{S}:=\{x \in \mathcal{X} \mid\|b(x)\| \neq 0\}$

- $x \rightarrow \theta(x)$ is a smooth positive function of $\mathcal{X}$ to $\mathbb{R}$, such that $\theta(x)\|\Delta(x)\|$ vanishes at the origin, and ensuring on $\mathcal{S} \backslash\{0\}$ the inequality $a(x)^{2}+\theta(x) b(x) \Delta(x) b(x)^{T}>0$

- $\sigma$ is a control parameter in $[0,1$,

- $\gamma: \mathcal{X} \rightarrow \mathbb{R}$ is defined by:

$$
\gamma(x):= \begin{cases}\frac{a(x)+\sqrt{a(x)^{2}+\theta(x) b(x) \Delta(x) b(x)^{T}}}{b(x) \Delta(x) b(x)^{T}} & \text { if } x \in \mathcal{S} \\ 0 & \text { if } x \notin \mathcal{S}\end{cases}
$$

As in Theorem 4.1, if the CLF satisfies the small control property, then the control is continuous at the origin. Moreover, if there exists some smooth function $\omega: \mathcal{X} \rightarrow \mathbb{R}^{+}$such that on $\mathcal{S} \backslash\{0\}$,

$$
\omega(x) b(x) \Delta(x) b(x)^{T}-a(x)>0
$$

then the control is smooth on $\mathcal{X}$ as soon as $\theta(x)\|\Delta(x)\|$ vanishes at the origin with:

$$
\theta(x):=\omega(x)^{2} b(x) \Delta(x) b(x)^{T}-2 a(x) \omega(x)
$$

Before giving the proof of Theorem 4.2, let us explain the ideas behind the construction of feedback (8). In the event function, the term $a(x)+b(x) k(m)$ is the time derivative of $V$ where $-\sqrt{a(x)^{2}+\theta(x) b(x) \Delta(x) b(x)^{T}}$ is the value of $\dot{V}$ if $k(x)$ is applied instead of $k(m)$. Therefore, right after an event, $e(x, m)$ necessarily takes a strictly positive value, more precisely, it equals $(1-\sigma) \sqrt{a(x)^{2}+\theta(x) b(x) \Delta(x) b(x)^{T}}$ and remains positive as long as $\dot{V} \leq-\sigma \sqrt{a(x)^{2}+\theta(x) b(x) \Delta(x) b(x)^{T}}$. Events will be more frequent with smaller $\sigma . \sigma=0$ means updating the control when $\dot{V}=0$. The second crucial tuning parameter of the control law is the choice of the function $\Delta$ that directly impacts the performance of the control as well as the frequency of events.

We next focus on homogeneous systems that gave rise to an important literature (see for instance [25], [26] and the references therein) and more recently for event-based approaches (mainly in [22], [18], [20]). We shortly recall few definitions: 
Definition 4.1: Let $r=\left(r_{1}, \ldots, r_{n}\right) \in \mathbb{R}_{\geq 0}^{n}$, and a set of coordinates $x=\left(x_{1}, \ldots, x_{n}\right)$.

- The family of dilations $\left(\delta_{\lambda}^{r}\right)_{\lambda>0}$ is defined by: $\delta_{\lambda}^{r}(x):=\left(\lambda^{r_{1}} x_{1}, \ldots, \lambda^{r_{n}} x_{n}\right), \forall \lambda>0$

- $V: \mathcal{X} \rightarrow \mathbb{R}$ is a $\delta^{r}$-homogeneous function of degree $d$ if: $\forall(x, \lambda) \in \mathcal{X} \times \mathbb{R}_{>0}, V\left(\delta_{\lambda}^{r}(x)\right)=\lambda^{d} V(x)$

- $h: \mathcal{X} \rightarrow \mathcal{X}$ is a $\delta^{r}$-homogeneous function of degree $d$ if: $\forall(x, \lambda) \in \mathcal{X} \times \mathbb{R}_{>0}, h\left(\delta_{\lambda}^{r}(x)\right)=\lambda^{d} \delta_{\lambda}^{r}(h(x))$

- A $\delta^{r}$-homogeneous norm $x \rightarrow\|x\|_{(r, \pi)}$ is a map defined by $\|x\|_{(r, \pi)}=\left(\sum_{i=1}^{n}\left|x_{i}\right|^{\frac{\pi}{r_{i}}}\right)^{\frac{1}{\pi}}$

To these classical definitions, and for the sake of brevity, we add:

Definition 4.2: Let an event-based controlled $\delta^{r}$-homogeneous system of degree $\left(d_{1}, d_{2}\right)$ denote a system of form (1) with an event-based feedback $(e, k)$ such that $f\left(\delta_{\lambda}^{r}(x), k\left(\delta_{\lambda}^{r}(m)\right)\right)=$ $\lambda^{d_{1}} f(x, k(m))$ and $e\left(\delta_{\lambda}^{r}(x), \delta_{\lambda}^{r}(m)\right)=\lambda^{d_{2}} e(x, m)$.

Property 4.3: Consider an event-based controlled $\delta^{r}$-homogeneous system of degree $\left(d_{1}, d_{2}\right)$, then the solution exists, is unique and such that $x\left(t ; \delta_{\lambda}^{r}\left(x_{0}\right)\right)=\delta_{\lambda}^{r}\left(x\left(\lambda^{d_{1}} t ; x_{0}\right)\right)$.

The proof trivially follows from the $\delta^{r}$-homogeneity of degree $d_{1}$ of $f(x, k(x))$ and using the relation $e\left(\Lambda^{r} x, \Lambda^{r} m\right)=\lambda^{d_{2}} e(x, m)$.

For homogeneous systems, Theorem 4.2 becomes the following. It is actually the event-based formulation of the smooth homogeneous control proposed in [27] that appears as a particular case of Theorem 4.2 with an appropriate choice of functions $\Delta(x)$ and $\omega(x)$.

Theorem 4.4: Assume that $f$, each $g_{i}$ and the CLF $V$ are $\delta^{r}$-homogeneous respectively of degree $d_{f}, d_{g_{i}}$, and $d_{V}$, with $\forall i \in\{1, \ldots, p\}, d_{g_{i}}<d_{f}$. Then, event-based feedback (8-9) with $\theta(x)$ as in (12), $\omega(x)=\nu\|x\|_{(r, \pi)}^{d_{f}-d_{V}}, \nu>0$ and $\delta_{i}(x)=\|x\|_{(r, \pi)}^{-2 d_{g_{i}}}$, is given by:

$$
k_{i}(x):=-\nu b_{i}(x)\|x\|_{(r, \pi)}^{d_{f}-2 d_{g_{i}}-d_{V}}
$$

For $\nu$ sufficiently large, this event-based feedback is such that:

1) the event-based controlled system is $\delta^{r}$-homogeneous of degree $\left(d_{f}, d_{V}+d_{f}\right)$

2) the event-based control is smooth and uniformly MSI

3) the CLF is strictly decreasing for all $x \in \mathcal{X} \backslash\{0\}$

Proof of Theorem 4.2: We begin the proof establishing $\gamma$ is smooth on $\mathcal{X} \backslash\{0\}$. For this, consider the algebraic equation:

$$
F(x, p):=b(x) \Delta(x) b(x)^{T} p^{2}-2 a(x) p-\theta(x)=0
$$


Note first that $p=\gamma(x)$ is a solution of (14) for all $x \in \mathcal{X}$. It is easy to prove that the partial derivative of $F$ with respect to $p$ is always strictly positive on $\mathcal{X} \backslash\{0\}$ :

$$
\frac{\partial F}{\partial p}:=2 b(x) \Delta(x) b(x)^{T} p-2 a(x)
$$

Indeed, when $\|b(x)\|=0$, equation (6) gives $\frac{\partial F}{\partial p}=-2 a(x)>0$ and when $\|b(x)\| \neq 0$, equation (10) gives $\frac{\partial F}{\partial p}=2 \sqrt{a(x)^{2}+\theta(x) b(x) \Delta(x) b(x)^{T}}>0$. Therefore $\frac{\partial F}{\partial p}$ never vanishes at each point of the form $\{(x, \gamma(x)) \mid x \in \mathcal{X} \backslash\{0\}\}$. Furthermore, $F$ is smooth w.r.t. $x$ and $p$ since so are $a, b, \theta$ and $\Delta$. Hence, using the implicit function theorem, $\gamma$ is smooth on $\mathcal{X} \backslash\{0\}$.

The decrease of the CLF is trivial to prove. Indeed, for each $x_{i} \neq 0, i \in \mathbb{N}$ :

$$
\frac{d V}{d t}\left(x_{i}\right)=\frac{\partial V}{\partial x}\left(x_{i}\right) f\left(x_{i}\right)+\frac{\partial V}{\partial x}\left(x_{i}\right) g\left(x_{i}\right) k\left(x_{i}\right)=-\sqrt{a\left(x_{i}\right)^{2}+\theta\left(x_{i}\right) b\left(x_{i}\right) \Delta\left(x_{i}\right) b\left(x_{i}\right)^{T}}<0
$$

With the updated control, the event function becomes strictly positive:

$$
e\left(x_{i}, x_{i}\right)=(1-\sigma) \sqrt{a\left(x_{i}\right)^{2}+\theta\left(x_{i}\right) b\left(x_{i}\right) \Delta\left(x_{i}\right) b\left(x_{i}\right)^{T}}>0
$$

Therefore, by smoothness of $V, f$ and $g$, it follows that $\frac{d V}{d t}\left(x\left(t ; x_{i}, t_{i}\right)\right)<0$ for all $t \in\left[t_{i}, t_{i+1}[\right.$, that is until the next event occurs. $t_{i+1}$ is necessarily bounded since, if not, $V$ should converge to a constant value where $\frac{d V}{d t}=0$, which is impossible. The event function precisely prevents this phenomena detecting $\frac{d V}{d t}$ is close to vanish and updates the control if it happens.

To prove that the event-based control is MSI, we have to prove that for any initial condition in a priori given set, the sampling intervals are bounded below. First of all, notice that events occur only when $e$ becomes negative (with $x \neq 0$ ) and therefore from equation (9), on $\{x \in \mathcal{X} \mid\|b(x)\|=0\} \backslash\{0\}:$

$$
e(x, m)=-a(x)-\sigma|a(x)|=(1-\sigma)|a(x)|>0
$$

It follows that there is no event on the set $\{x \in \mathcal{X} \mid\|b(x)\|=0\} \cup\{0\}$. We hence restrict the study to the set $\{x \in \mathcal{X} \mid \beta(x) \neq 0\} \backslash\{0\}$ where $\theta$ and the $\delta_{i}$ 's are strictly positive by assumption. Let us rewrite the time derivative of the CLF along the trajectories:

$$
\begin{aligned}
\frac{d V}{d t}(x) & =a(x)+b(x) k(m) \\
& =-\sqrt{a(x)^{2}+\theta(x) b(x) \Delta(x) b(x)^{T}}+b(x)(k(m)-k(x))
\end{aligned}
$$


Let us define for $m \in \mathcal{S}$, the level $\vartheta_{m}:=V(m)$ and the set $\mathcal{V}_{\vartheta_{m}}:=\left\{x \in \mathcal{X} \mid V(x) \leq \vartheta_{m}\right\}$. Then in equation (15), it follows from the choice of the event function that $x$ belongs to $\mathcal{V}_{\vartheta_{x}} \subset \mathcal{V}_{\vartheta_{m}}$. Note that if $m$ belongs to $\mathcal{S}$, this is not necessarily the case for $x$ that can escape from this set. First see that for $t=t_{i}$ (recall the $t_{i}$ 's denote the time instant when an event occurs), $x=m$ and therefore, since i) $\theta(x)$ is such that $a(x)^{2}+\theta(x) b(x) \Delta(x) b(x)^{T}>0$ for all nonzero $x$ in $\mathcal{S}$, and ii) $a(x)$ is necessarily nonzero on the frontier of $\mathcal{S}$ (except possibly at the origin):

$$
\begin{aligned}
\frac{d V}{d t}(x) & =-\sqrt{a(m)^{2}+\theta(m) b(m) \Delta(m) b(m)^{T}} \\
& \leq-\inf _{\substack{m \in \mathcal{S} \\
\text { s.t. } V(m)=\vartheta_{m}}} \sqrt{a(m)^{2}+\theta(m) b(m) \Delta(m) b(m)^{T}}=:-\chi\left(\vartheta_{m}\right)<0
\end{aligned}
$$

Considering now the second time derivative of the CLF:

$$
\ddot{V}(x)=\left(\frac{\partial a}{\partial x}(x)+k(m)^{T} \frac{\partial b^{T}}{\partial x}(x)\right)(f(x)+g(x) k(m))
$$

By continuity of all the involved functions, both terms can be bounded for all $x \in \mathcal{V}_{\vartheta_{m}}$ by the following upper bounds $\varrho_{1}\left(\vartheta_{m}\right)$ and $\varrho_{2}\left(\vartheta_{m}\right)$ :

$$
\begin{aligned}
& \varrho_{1}\left(\vartheta_{m}\right):=\sup _{\substack{m \in \mathcal{S} \text { s.t. } V(m)=\vartheta_{m} \\
x \in \mathcal{V}_{\vartheta_{m}}}}\left\|\frac{\partial a}{\partial x}(x)+k(m)^{T} \frac{\partial b^{T}}{\partial x}(x)\right\| \\
& \varrho_{2}\left(\vartheta_{m}\right):=\sup _{\substack{m \in \mathcal{S} \text { s.t. } V(m)=\vartheta_{m} \\
x \in \mathcal{V}_{\vartheta_{m}}}}\|f(x)+g(x) k(m)\|
\end{aligned}
$$

Therefore, $\dot{V}$ is strictly negative at any event instant $t_{i}$ and can not vanish until a certain time $\underline{\tau}\left(\vartheta_{m}\right)$ is elapsed and this minimal sampling is only depending on the level $\vartheta_{m}$ of the CLF in $m$ (and hence of the radius of any ball inside):

$$
\underline{\tau}\left(\vartheta_{m}\right) \geq \frac{\chi\left(\vartheta_{m}\right)}{\varrho_{1}\left(\vartheta_{m}\right) \varrho_{2}\left(\vartheta_{m}\right)}>0
$$

which ends the proof, the event-based feedback (8-9) is semi-uniformly MSI. 
To prove the continuity of $k$ at the origin, we only need to consider the points in $\mathcal{S}$ since we already have $k(x)=0$ if $\|b(x)\|=0$. We have:

$$
\begin{aligned}
\|k(x)\| & \leq \frac{|a(x)|+\sqrt{a(x)^{2}+\theta(x) b(x) \Delta(x) b(x)^{T}}}{b(x) \Delta(x) b(x)^{T}}\left\|\Delta(x) b(x)^{T}\right\| \\
& \leq \frac{2|a(x)|}{b(x) \Delta(x) b(x)^{T}}\left\|\Delta(x) b(x)^{T}\right\|+\frac{\sqrt{\theta(x)}}{\sqrt{b(x) \Delta(x) b(x)^{T}}}\left\|\Delta(x) b(x)^{T}\right\| \\
& \leq \frac{2|a(x)|}{b(x) \Delta(x) b(x)^{T}}\left\|\Delta(x) b(x)^{T}\right\|+\sqrt{\theta(x)\|\Delta(x)\|}
\end{aligned}
$$

With the small control property, for any $\varepsilon>0$, there is $\mu>0$ such that for any $x \in \mathcal{B}(\mu) \backslash\{0\}$, there exists some $u$ with $\|u\| \leq \varepsilon$ such that $a(x)+b(x) u<0$ and therefore $|a(x)|<\|b(x)\| \varepsilon$. It follows:

$$
\|k(x)\| \leq \frac{2 \varepsilon\|b(x)\|\left\|\Delta(x) b(x)^{T}\right\|}{b(x) \Delta(x) b(x)^{T}}+\sqrt{\theta(x)\|\Delta(x)\|}
$$

Since the function $\left(v_{1}, v_{2}\right) \rightarrow \frac{\left\|v_{1}\right\|\left\|v_{2}\right\|}{v_{1}^{T} v_{2}}$ is continuous w.r.t its two variables at the origin where it equals 1 , since $\theta$ and $\Delta$ are also continuous, since $\theta(x)\|\Delta(x)\|$ vanishes at the origin, for any $\varepsilon^{\prime}$, there is some $\mu^{\prime}$ such that $\forall x \in \mathcal{B}\left(\mu^{\prime}\right) \backslash\{0\},\|k(x)\| \leq \varepsilon^{\prime}$ which ends the proof of continuity.

Finally, with $\theta$ as in (12), the control becomes $k_{i}(x)=-b_{i}(x) \delta_{i}(x) w(x)$ which is obviously smooth on $\mathcal{X}$.

Proof of Theorem 4.4: Take $\nu$ such that:

$$
\nu>\sup _{x \in\left\{x \in \mathcal{X} \mid\|x\|_{(r, \pi)}=1, a(x) \geq 0\right\}} \frac{a(x)}{\sum_{i=1}^{p} b_{i}(x)^{2}\|x\|_{(r, \pi)}^{d_{f}-2 d_{g_{i}}-d_{V}}}
$$

As in [27], $k$ is $\delta^{r}$-homogeneous of degree $d_{f}-d_{g}$ and the system is therefore $\delta^{r}$-homogeneous of degree $d_{f}$. In addition $e$ clearly satisfies $e\left(\delta_{\lambda}^{r}(x), \delta_{\lambda}^{r}(m)\right)=\lambda^{d_{V}+d_{f}} e(x, m)$. Item 1 of Theorem 4.4 therefore holds.

On $\left\{x \in \mathcal{X} \mid\|x\|_{(r, \pi)}=1, a(x) \geq 0\right\}$, equation (17) gives $\omega(x) b(x) \Delta(x) b(x)^{T}-a(x)>0$. Since the right-hand side of the inequality is $\delta^{r}$-homogeneous of degree $d_{V}+d_{f}$, the same inequality holds for all $x \neq\{0\}$ such that $a(x) \geq 0$. Using (6), it follows that this inequality is valid for $x$ in $\mathcal{S} \backslash\{0\}$. Therefore, equation (11) holds with the above choice of $\omega$. Since $\theta$ given by equation (12) is homogeneous of degree $2 d_{f}$ and using the property $d_{g_{i}}<d_{f}$ that holds for all $i \in\{1, \ldots, p\}$, it follows that $\lim _{x \rightarrow 0} \theta(x)\|\Delta(x)\|=0$. Thanks to Theorem 4.2, item 3 holds and the control is smooth and semi-uniformly MSI. 
To finish the proof, remains to establish that the event-based feedback is uniformly MSI. For this, we invoke the homogeneity of the Lyapunov level sets $\mathcal{V}_{\vartheta_{m}}$ together with the relation $x\left(t ; \delta_{\lambda}^{r}\left(x_{0}\right)\right)=\delta_{\lambda}^{r}\left(x\left(\lambda^{d_{1}} t ; x_{0}\right)\right)$, that gives for all $\vartheta_{m}>0, \underline{\tau}\left(\vartheta_{m}\right)=\underline{\tau}(1)$ that ends the proof of item 2 .

\section{EXAMPLES}

\section{A. Linear time invariant systems}

Consider the linear time-invariant system $\dot{x}=A x+B u$. Take $P$, a positive definite matrix solution of the Riccati equation $P A+A^{T} P-4 \varepsilon P B B^{T} P=-P$. Then $V(x):=x^{T} P x$ is a CLF for the system since for all $x, u=-2 \varepsilon B^{T} P x$ renders $\dot{V}$ strictly negative for $x \neq 0$. Since $a(x)=x^{T}\left(P A+A^{T} P\right) x, b(x)=2 x^{T} P B$ and $\beta(x)=4 x^{T} P B B^{T} P x$, the Riccati takes the form of equation (11) with $\omega(x):=\varepsilon$ and $\delta_{i}:=1$ for all $i \in\{1, \ldots, p\}$. Therefore, taking $\theta(x)$ according to (12), the control is smooth everywhere and linear: $k(x)=-\varepsilon b(x)^{T}$.

\section{B. Nonlinear system}

We consider next the nonlinear system proposed in [21]:

$$
\begin{aligned}
& \dot{x}_{1}=-x_{1}^{3}+x_{1} x_{2}^{2} \\
& \dot{x}_{2}=x_{1} x_{2}^{2}-x_{1}^{2} x_{2}+u
\end{aligned}
$$

that admits $V(x)=\frac{1}{2} x_{1}^{2}+\frac{1}{2} x_{2}^{2}$ as CLF with $a(x)=-x_{1}^{4}+x_{1} x_{2}^{3}, b(x)=x_{2}$. We first take $\delta_{i}:=1$ for all $i \in\{1, \ldots, p\}, \theta(x)=b(x)^{2}$ and $\sigma=0.9$. The time evolution of $x, V(x), k(x)$ and the event function $e$ is depicted in Figure 1 for $x_{0}=(0.1,0.4)$.

Considering $\omega(x)=x_{1} x_{2}+\frac{1}{2} x_{1}^{2}+\frac{1}{2} x_{2}^{2}$ satisfies inequality (11) on $\mathcal{S} \backslash\{0\}$ :

$$
\omega(x) b(x) \Delta(x) b(x)^{T}-a(x)=\frac{1}{2} x_{1}^{2} x_{2}^{2}+\frac{1}{2} x_{2}^{4}+x_{1}^{4}>0
$$

it follows that taking $\theta(x)$ as in (12), the control is smooth everywhere. The resulting trajectory with $\sigma=0.9$ is represented in Figure 1 .

In both cases, event occurs twice in the $40 \mathrm{~s}$ of time horizon (the initial event is included). With the discontinuous control, the convergence is faster but the control value is also almost ten times larger. When compared to [21] with the same initial condition and the parameter $\sigma=0.9$ (the higher $\sigma$ is, less frequent events are), 117 events are obtained with a maximum value of 

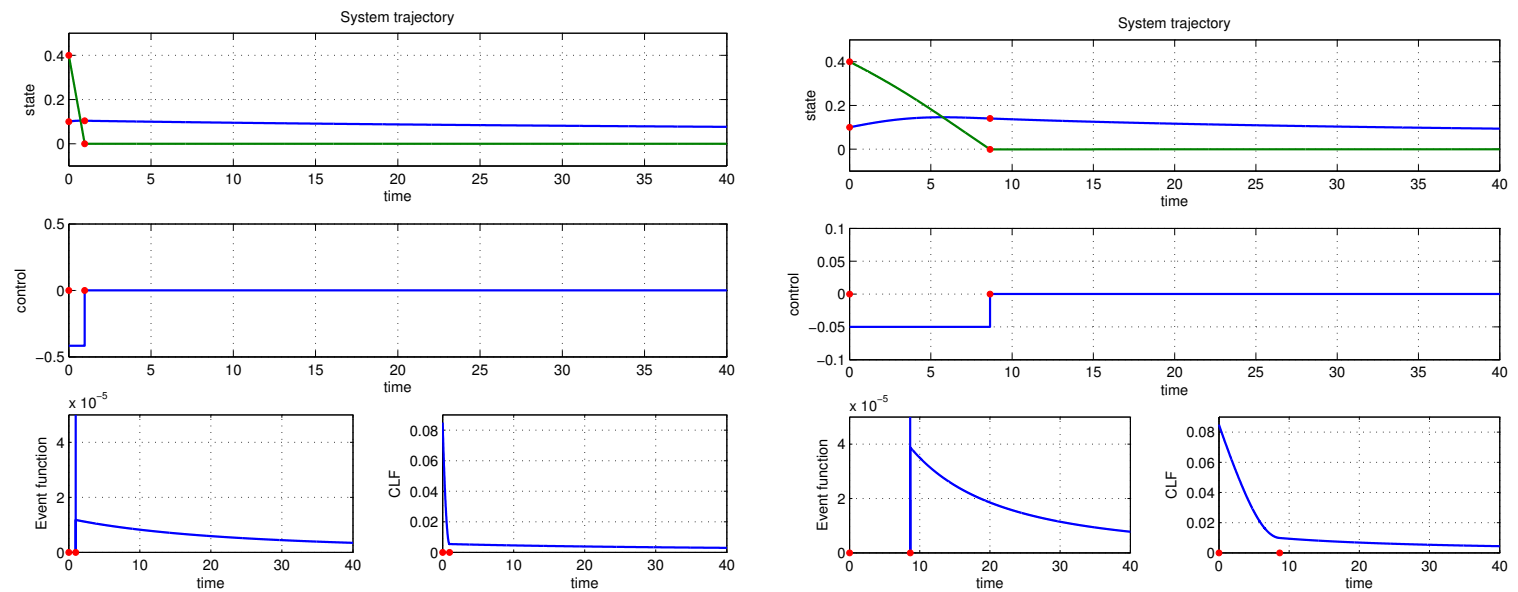

Fig. 1. System (18) under non smooth (left) and smooth (right) event-based control. The red dots represents the events.

the control power of -0.08 , that is higher than the -0.05 with the smooth control. Moreover, the decrease of the CLF is slower than with the smooth and non smooth control proposed here as illustrated in Figure 2. In this example, with the same system, the same CLF, the proposed control and control update policy seems more efficient (faster convergence, smaller control, fewer events).

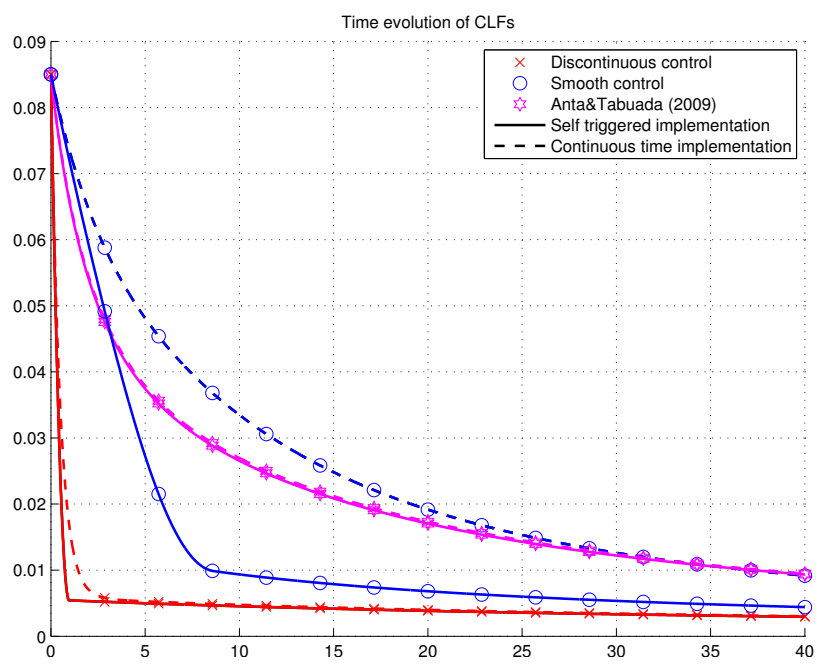

Fig. 2. Time evolution of the CLF using the non smooth and smooth version of the proposed control law compared to [21] and a continuous time implementation. 


\section{CONCLUSION}

In this paper, we proposed an extension of the universal formula for feedback stabilization to event-based controlled systems. A modification of the original formula is necessary to ensure that there is a minimal sampling time between two consecutive events avoiding zeno phenomena like accumulation points. As in the original work, if the Control Lyapunov Function fulfils the small control property, then the control is continuous at the origin. With additional homogeneity assumptions, the control can be proved to be smooth everywhere and the minimal inter-sampling time bounded below for all initial conditions.

\section{REFERENCES}

[1] K. Åström and B. Wittenmark, Computer Controlled Systems, 3rd Edition. Prentice Hall, 1997.

[2] A. Cervin, D. Henriksson, B. Lincoln, J. Eker, and K. Arzen, "How does control timing affect performance? analysis and simulation of timing using jitterbug and TrueTime," IEEE Control Systems Magazine, vol. 23, no. 3, pp. 16-30, 2003.

[3] P. Hsu and S. Sastry, "The effect of discretized feedback in a closed loop system," in Proc. of the IEEE Conference on Decision and Control (CDC), vol. 26, 2007, pp. 1518-1523.

[4] D. Nešić and A. Teel, "A framework for stabilization of nonlinear sampled-data systems based on their approximate discrete-time models," IEEE Trans. on Automatic Control, vol. 49, no. 7, pp. 1103-1122, 2004.

[5] D. Nešić and L. Grüne, "Lyapunov-based continuous-time nonlinear controller redesign for sampled-data implementation," Automatica, vol. 41, no. 7, pp. 1143-1156, 2005.

[6] F. H. Clarke, Y. S. Ledyaev, E. D. Sontag, and A. Subbotin, "Asymptotic controllability implies feedback stabilization," IEEE Trans. on Automatic Control, vol. 42, no. 10, pp. 1394-1407, 1997.

[7] N. Marchand and M. Alamir, NonLinear Control in the Year 2000. Londres, UK: Springer Verlag, Dec. 2000, vol. 2, ch. Asymptotic controllability implies continuous discrete time feedback stabilizability, pp. 63-79.

[8] K.-E. Årzén, "A simple event-based PID controller," in Preprints of the 14th World Congress of IFAC, Beijing, P.R. China, 1999.

[9] J. Sandee, W. Heemels, and P. van den Bosch, "Event-driven control as an opportunity in the multidisciplinary development of embedded controllers," in Proc. of the IEEE American Control Conference (ACC), 2005, pp. 1776-1781.

[10] S. Durand and N. Marchand, "Further results on event-based PID controller," in Proc. of the European Control Conference (ECC), 2009.

[11] J. Sánchez, M. Guarnes, S. Dormido, and A. Visioli, "Comparative study of event-based control strategies: An experimental approach on a simple tank," in Proc. of the European Control Conference (ECC), 2009.

[12] J. Sánchez, M. Guarnes, and S. Dormido, "On the application of different event-based sampling strategies to the control of a simple industrial process," Sensors, vol. 9, pp. 6795-6818, 2009.

[13] K. Åström and B. Bernhardsson, "Comparison of Riemann and Lebesque sampling for first order stochastic systems," in Proc. of the IEEE Conference on Decision and Control (CDC), 2002.

[14] W. Heemels, J. Sandee, and P. van den Bosch, "Analysis of event-driven controllers for linear systems," International journal of control, vol. 81, pp. 571-590, 2009. 
[15] J. Lunze and D. Lehmann, "A state-feedback approach to event-based control," Automatica, vol. 46, pp. 211-215, 2010.

[16] M. Donkers and W. Heemels, "Output-based event-triggered control with guaranteed $\mathcal{L}_{\infty}$-gain and improved eventtriggering," in Proc. of the IEEE Conference on Decision and Control (CDC), 2010.

[17] A. Eqtami, D. Dimarogonas, and K. Kyriakopoulos, "Event-triggered control for discrete-time systems," in Proc. of the IEEE American Control Conference (ACC), 2010, pp. 1776-1781.

[18] M. J. Mazo, A. Anta, and P. Tabuada, "On self-triggered control for linear systems: Guarantees and complexity," in Proc. of the European Control Conference (ECC), 2009.

[19] M. Velasco, P. Martí, and E. Bini, "On Lyapunov sampling for event-driven controllers," in Proc. of the IEEE Conference on Decision and Control (CDC), 2009.

[20] P. Tabuada, "Event-triggered real-time scheduling of stabilizing control tasks," IEEE Trans. on Automatic Control, vol. 52, no. 9, pp. 1680-1685, 2007.

[21] A. Anta and P. Tabuada, "Self-triggered stabilization of homogeneous control systems," in Proc. of the IEEE American Control Conference (ACC), 2008, pp. 4129-4134.

[22] — - "To sample or not to sample: Self-triggered control for nonlinear systems," IEEE Trans. on Automatic Control, vol. 55, pp. 2030 - 2042, 2010.

[23] E. D. Sontag, "Clocks and insensitivity to small measurement errors," ESAIM Control, Optimisation and Calculus of Variations, vol. 4, pp. 537-557, 1999.

[24] — Mathematical control theory, deterministic finite dimensional systems, 2nd ed. Springler Verlag, 1998.

[25] L. Faubourg and J. Pomet, "Control Lyapunov functions for homogeneous Jurdjevic-Quinn systems," ESAIM: Control, Optimisation and Calculus of Variations, vol. 5, pp. 293-311, 2000.

[26] L. Grüne, "Homogeneous state feedback stabilization of homogeneous systems," in Proc. of the IEEE Conference on Decision and Control (CDC), vol. 4. IEEE, 2000, pp. 3409-3414.

[27] J. Tsinias, "Remarks on feedback stabilizability of homogeneous systems," Control Theory and Advanced Technology, vol. 6, pp. 533-542, 1990.

[28] M. Jankovic, R. Sepulchre, and P. Kokotovic, "CLF based designs with robustness to dynamic input uncertainties," Systems and Control Letters, vol. 37, no. 1, pp. 45-54, 1999. 